\title{
"El caso de Guatemala": \\ doctrina y praxis de la delegación guatemalteca en el I Congreso Anticomunista Latinoamericano
}

\author{
"The Case of Guatemala": \\ Doctrine and Praxis of the Guatemalan \\ Delegation in I Latin American \\ Anti-Communist Congress
}

\author{
Juan Carlos Vázquez Medeles"
}

RESUMEN: en mayo de 1954 se efectuó el I Congreso contra la Intervención Soviética en América Latina. El discurso anticomunista desplegado, manifiesto en su doctrina y praxis, fue el preámbulo de la invasión liberacionista que depuso al presidente de Guatemala Jacobo Árbenz Guzmán. Estudio pionero en la temática, para este artículo se revisaron exhaustivamente los documentos de la Agencia Central de Inteligencia y los textos de autores que han estudiado el tema. Se privilegia tanto el ejercicio políitico de estos agentes como la consolidación de sus ideas y materiales en el devenir histórico de este país. Se concluye que su protagonismo se estableció como un hito en los entrecruces e interacciones con los sujetos y grupos latinoamericanos, afines a su ideología, en el periodo que se insertó la región en el conflicto ideológico global.

Palabras ClaVE: Anticomunismo; Guatemala; Representaciones; Congreso Anticomunista.

ABSTRACT: The article describes the participation of the Guatemalan delegation in the 1st Congress against Soviet Intervention in Latin America, held in May 1954. The objective is to articulate its anti-communist discourse, manifested in its doctrine and praxis, as a preamble to the liberationist invasion that deposed to President Jacobo Árbenz Guzmán. As a pioneering study on the subject, the documents of the Central Intelligence Agency and the texts of authors who have approached the topic were exhaustively reviewed. As a result, the political exercise of these agents is accentuated, as well as the consolidation of their ideas and the materials in the historical development or their country. It is added that their protagonism was established as a milestone in the intercrossings and interactions with Latin America subjects and groups, related to its ideology, in the period was inserted into the global ideological conflict.

KEY wORDS: Anticommunism; Guatemala; Representations; Anticommunist Congress.

Recibido: 11 de diciembre de 2019

Aceptado: 21 de agosto de 2020

Becario del Centro de Investigaciones sobre América Latina y el Caribe (CIALC-UNAM), asesorado por el doctor Mario Vázquez Olivera (vazquezjc@gmail.com). 
PROLEGÓMENOS A UN PROYECTO DOMINANTE

ANTICOMUNISTA

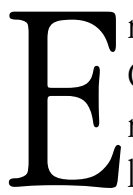

n diciembre de 2015, el párroco del templo de la Merced de la Ciudad de Guatemala anunció que se ascendería al grado de general del Ejército a la imagen devocional del Jesús Nazareno. Ante la reacción y crítica a la polémica declaración, el presidente interino Alejandro Maldonado Aguirre se desvinculó del hecho que enfatizaba la permanencia del anticomunismo en la administración y en su figura. La situación evidenció la estrecha relación entre la Iglesia, el anticomunismo y el Ejército de Liberación Nacional en la construcción del Estado guatemalteco con esta perspectiva ideológica. Para comprender la irrupción del anticomunismo es necesario analizarlo como una doctrina y una praxis que evidencia las relaciones de poder que se entrelazaron en la acción colectiva de distintos grupos sociales.

Este trabajo estudia la participación de los delegados guatemaltecos en el I Congreso contra la Intervención Soviética en América Latina. El análisis considera la noción de hegemonía política de Antonio Gramcsi, con la finalidad de establecer la articulación de las formas culturales, sociales y políticas que permitieron el ejercicio de la dominación de los anticomunistas guatemaltecos y colocarse como dirigentes de la voluntad colectiva (Gramsci 1981: 107). Para lograrlo, ordenaron una estructura ideológica que desplegó una organización material y estableció una dirección moral con la aquiescencia de otros grupos, articulada a través de símbolos, normas y principios. Para abordar este anticomunismo, me ceñiré a la propuesta de Patto Sá Motta (2000), que considera las siguientes matrices: el catolicismo, el nacionalismo y el liberalismo, aunque este último, en Guatemala, tuvo rechazo implícito en los preceptos religiosos y el imperante desarrollismo de la época.

Esta investigación tiene como objetivo analizar la manera en que se articuló el discurso anticomunista en Guatemala, basado en la doctrina y la praxis que permitió establecer una hegemonía política. Con base en esta premisa, los participantes del Congreso se consideran como los agentes que moldearon el paradigma e ideario filosófico operativo que se enquis- 
tó en dicho país, y con ello se legitimó el uso de la violencia en su lucha contra el comunismo y cualquier idea que contrariase su concepción de democracia y libertad. Esto tuvo repercusiones devastadoras en la población y en el propio Estado, al debilitar tanto el marco institucional como el sistema político. Los representantes guatemaltecos dentro del Congreso defendieron su proyecto político y la imperativa destitución del gobierno en curso. El ejercicio de su doctrina dio lugar a establecer interconexiones políticas que les permitieron consolidar el ascenso al poder, a través del golpe contra el presidente Jacobo Árbenz Guzmán por parte del Ejército de Liberación Nacional, el trabajo logístico que les facilitó la Agencia Central de Inteligencia (CIA) y los entrecruces trasnacionales que lograron concretar.

Por ello, afirmo que, como se verá más adelante, los resultados de la reunión fueron exitosos, conforme a los objetivos del plan PBSUCCESS que se ciñeron a las actividades encaminadas a deponer a dicho gobierno, frente a los análisis que denuestan su impacto inmediato y regional (Jiménez 2017). Si bien la participación de la delegación guatemalteca es el eje de este trabajo, el contexto en el que se dio permite entender su trascendencia más allá de la región centroamericana, pues se enmarca en los procesos de globalización que menciona Víctor Hugo Acuña, y que son vistos como "el conjunto de procesos crecientes de interconexión y de interdependencia entre las distintas partes del planeta, en los que fluyen personas, ideas y objetos" (Acuña 2015: 14). Sin embargo, a diferencia de algunos estudios que se adhieren al giro global, como en el caso de Pettinà, y que esquematizan estos procesos (Pettinà 2018: 86), esta investigación pondera el desarrollo de las actividades y las ideas que dieron forma tanto al Congreso referido como a sus resultados materiales y políticos. Para ello, se consideraron los estudios más recientes que refieren al anticomunismo, sus conexiones e interacciones en las organizaciones y sujetos protagonistas de este Congreso y los que lo sucedieron. Tal es el caso de Cañón Voirín, que expone el proceso de creación de la Confederación Interamericana de Defensa del Continente (CIDC) y presenta un breve bosquejo de los Congresos realizados por esta organización (Cañón 2017). En esta misma línea, se ubican la ponencia presentada por Bohos- 
lavsky y Broquetas (2018) y el trabajo de Marcelo Casals, quien resalta la participación de la delegación chilena y subraya la participación de Sergio Fernández Larraín (Casals 2019). Por último, Vázquez Medeles (2020) aborda el caso guatemalteco desde las reuniones organizadas por la CIDC hasta las realizadas por la Confederación Anticomunista Latinoamericana en las décadas de los setenta y ochenta.

En este artículo se aborda, en primer lugar, el proceso de organización de la reunión celebrada en México en mayo de 1954. Posteriormente, la participación de los delegados guatemaltecos y la influencia política que les permitió estructurar su concepción de anticomunismo. Para finalizar, se presenta la publicación El libro negro del comunismo en Guatemala (Secretaría General de la Comisión Permanente del Primer Congreso Contra la Intervención Soviética en América Latina 1954), como parte del trabajo que prosiguió a las actividades del comité organizador y compromisos asumidos por los participantes en el evento.

\section{LOS DERROTEROS ORGANIZATIVOS DEL CONGRESO CONTINENTAL ANTICOMUNISTA}

El anticomunismo en Guatemala fue adoptado como ideología por la derecha política, como doctrina permeó en las elites políticas y económicas para enfrentar a su adversario. En un momento, lo hizo contra los gobiernos democráticos de Juan José Arévalo Bermejo (1945-1951) y Jacobo Árbenz Guzmán (1951-1954) y, posteriormente, combatió a las organizaciones revolucionarias y el pensamiento en que basaron su proyecto político. La participación de una delegación en el Congreso Anticomunista de 1954 les permitió actuar como portadores simbólicos de esta ideología y operaron como sujetos cohesionadores de la acción social que se tradujo en la consolidación hegemónica de una colectividad dominante.

Es importante considerar el contexto histórico de este Congreso, se trata del periodo de la posguerra, en el que las potencias hegemónicas, Estados Unidos y la Unión de Repúblicas Soviéticas Socialistas (URSS), dinamizaron la política mundial y reorganizaron las relaciones económicas al enfrentarse en un nuevo conflicto llamado "Guerra Fría". En este escena- 
rio, los guatemaltecos vivieron su propio proceso (1944 a 1954), periodo que representó un punto de intersección entre la política interior y los intereses hegemónicos estadounidenses que atrajeron hacía América Latina el conflicto ideológico mundial. La administración del presidente Dwight D. Eisenhower (1953-1961) buscó coartar este proceso bajo un discurso proteccionista de sus intereses económicos, y la aplicación de medidas preventivas ante el advenimiento de ideas comunistas en el continente. El 9 de septiembre de 1953 autorizó a la CIA un presupuesto de 270 mil dólares para el desarrollo de la operación encubierta denominada PBSUCCESS. En ella se combinarían acciones sicológicas, económicas, diplomáticas y paramilitares para ponderar el trabajo de propaganda, "destinada a desacreditar a un enemigo y construir apoyo para los aliados" (Cullather 1994: 26). Se le asignó el 9\% del total de recursos a la guerra sicológica y acciones políticas (Freedom of Information Act-Electronic Reading Room) (FOIA/ERR), Budget Summary (PBFORTUNE 1953: 1) que serían desarrolladas y evaluadas por el centro de operaciones KugOwn, criptónimo de Propaganda (Departament of State 2003: XVI).

Una de sus acciones consistió en brindar apoyo para la realización de un Congreso. El objetivo era crear apoyo popular para la lucha contra los comunistas en Guatemala, "sin mostrar la mano americana" (FOIA/ERR, Project PBSUCCESS, RYBAT/PBSUCCESS 1954: 174). La Agencia aprovechó el interés que tenía el presidente del Frente Popular Anticomunista Mexicano (FPAM), Jorge Prieto Laurens, por organizar dicho evento y así crear una fachada totalmente regional. Un documento de la CIA con el programa del "Congreso de los Vencedores del Comunismo" muestra que, para su realización, se planteó la necesidad de contar con asistentes con prestigio político e intelectual y que se debería exponer el caso guatemalteco (FOIA/ERR, Congreso Panamericano Anticomunista 1954: 1-7). Se calculó que el costo de insumos era de 25900 dólares (FOJA/ERR, Hemisphere Conference in México, abril 1954: 10) e informaron que había promesas de apoyo económico desde la sede que les facilitaría el gobernador del Estado de Morelos, México, Rodolfo López de Nava; pasajes de avión de la American Airlines y hasta 200 mil pesos que fueron ofrecidos por el presidente mexicano Adolfo Ruiz Cortines. Además, se promovía una solicitud de ayuda a la 
United Fruit Company (UFCO) y a los senadores republicanos por el Estado de Wisconsin de Estados Unidos, Alexander Wiley y Joseph McCarthy (FOIA/ERR, Anti-Communism in Mexico- Specific- Frente Popular Anti-Comunista de México 1954: 1-3). Para ese momento, no contaban físicamente con el dinero que, en un segundo cálculo, estimaron en 100 mil dólares, casi cuatro veces más que la valoración previa. Ante la desconfianza que manifestó el Centro de operaciones KUGOWN hacia los miembros del FPAM (FOIA/ERR, Congreso Panamericano Anticomunista 1954: 1-7), estos últimos comisionaron al guatemalteco José Calderón Salazar ${ }^{1}$ para entrevistarse con los organizadores del ahora llamado "Congreso de los Combatientes Progresivos contra el Comunismo" y le proporcionaron 10 mil dólares bajo la condición de asegurar la participación de los grupos de guatemaltecos exiliados en México y que el tema de su país se examinara durante los dos últimos días del encuentro (FOIA/ERR, Mexico City Congress 1954: 1-6).

Al aceptar estos requerimientos, por parte de los organizadores, se eligieron cuatro emisarios para realizar una gira de promoción en diferentes países de la región, uno fue el diputado guatemalteco José Luis Arenas Barrera (el Tigre de Ixcán), ${ }^{2}$ quien estuvo acompañado por su compatriota,

1 Calderón Salazar fue activo periodista desde el régimen de Jorge Ubico. En 1941 fue nombrado archivero en la Embajada de Guatemala en México y para 1944 era el primer secretario de la misma (Archivo Histórico Genaro Estrada-Acervo Histórico Diplómatico) (AHGE-AHD), III/323 (728.1)/15, fs. 1-6). Sus ideas nacionalistas y su autoidentificación como criollo veían la necesidad de asimilación de esta cultura por parte del indígena. Mantuvo relación con la Falange en Guatemala, publicó en su revista Amanecer y, en 1944, dirigió la revista católica Verbum. Su ferviente catolicismo se reflejó en su pluma, como periodista utilizó el seudónimo de Guzmán de Alfarache, tomado de las obras del escritor sevillano Mateo Alemán, escritas a finales del siglo XVI, que si bien pertenecen al género de la novela picaresca, contienen un fuerte discurso moral. En 1947 escribió el libro Guatemala bajo el signo rojo, año en que inició su exilio en México. En 1954, junto a Roberto Gómez de León, encabezó el Comité de Exiliados Guatemaltecos Anticomunistas en México (CEGAM), donde se aglutinó al Frente Anticomunista de Guatemala en el Exilio (FAGE) y el Comité de Estudiantes Universitarios Anticomunistas Guatemaltecos en el Exilio (CEUAGE). Se considera el ideólogo de la Oposición Organizada, posteriormente, Movimiento de Liberación Nacional, inspirado en el nacional-catolicismo.

2 Arenas Barrera era diputado por la Ciudad de Guatemala, afiliado al Partido Unificación Anticomunista (PUA), del cual era fundador. En el Congreso conformó el grupo 
el abogado Luis Coronado Lira, el ingeniero Manuel Salazar Arce y el periodista Florencio Ávila Sánchez del FPAM, estos últimos de origen mexicano (FOIA/ERR, Travel Report J. L. Arenas, Manuel Salazar 1954: 1-10). Durante el viaje, realizado en el mes de mayo de 1954, contactaron al presidente venezolano Marcos Evangelista Pérez Jiménez para que les facilitara recursos, asimismo, visitaron diversos medios informativos de México, Cuba, República Dominicana, Panamá, Costa Rica, Nicaragua y Honduras (FOIA/ ERR, Cable Requesting Coverage of José Luis Arenas visit to Caracas 1954: 1).

Entre los preparativos para el Congreso hubo la intención de proyectar a Coronado Lira, ${ }^{3}$ pues lo consideraron con vasta experiencia política y con buenos resultados en las actividades coordinadas entre los exiliados y la CIA. En el discurso que se le preparó desde la Agencia: "Interven-

autodenominado los "Doce Apóstoles", junto a los miembros de su partido, el Partido Unión Demócratica, el Partido Independiente Anticomunista de Occidente y algunos otros diputados, que se convirtieron en la oposición de los gobiernos revolucionarios. Al volcarse al movimiento de Liberación perdió la curul en el Congreso de la República. Era dueño de la finca La Perla, ubicada en San Gaspar Chajul, Quiché. El crecimiento de su propiedad se debe al despojo de tierras a las comunidades ixiles asentadas en el área. Se dedicaba principlamente al cultivo de café y se convirtió en una de las plantaciones más importantes de la región.

3 Durante el gobierno de Ubico, Coronado Lira era un prominente masón y junto a su socio Carlos Salazar Gatica representaba a compañías alemanas. Fue de los firmantes del Memorial de los 311, por el cual exigieron a Jorge Ubico restablecer las garantías constitucionales. Para las elecciones de 1944, encabezó el Frente Nacional Democrático (FND) que postuló a Adrián Recinos a la presidencia. Al quedar frustradas sus intenciones políticas y después de permanecer unos meses preso, se exilió en México, Honduras y El Salvador, fue de los primeros detractores que buscaron concretar un movimiento revolucionario, ayudado por Anastasio Somoza García y Rafael Leónidas Trujillo. En el Salvador dirigió el periódico El Combate, órgano de difusión del FAGE, así como la radiodifusora Cristal. A partir de las emisiones que realizaron, se publicó el folleto: La Tribuna de la libertad: voz de los guatemaltecos en el destierro. Del 13 al 27 de marzo de 1954 viajó Caracas, Venezuela, junto a Luis Valladares y Aycinema y Salazar Gatica, que representó a la Oposición Organizada, y para manifestarse en la reunión de la Organización de Estados Americanos (OEA). Finalmente la Agencia lo vio con recelo ante su acercamiento con Juan Domingo Perón, en Buenos Aires, Argentina, mientras realizaban la gira promocional del evento, pues sospechaban de que le solicitó apoyo, enviado directamente por Castillo Armas (FOI/ERR: Revolutionary plans of Luis Coronado Lira, a guatemalan in exile 1948: 1 ; visit of the delegates of the organized opposition [Guatemalan] to Caracas 1954: 1-12; Coy 2016). 
ción soviética en Guatemala", se argumentó la legitimidad de su lucha y por ello la necesidad de solicitar asistencia de otras naciones. Básicamente, enfatizaba el carácter extranjero de la penetración ideológica marxista y la contribución del Partido Guatemalteco del Trabajo (PGT) para que esto sucediera. Aunque parece discordante, su constructo social nacionalista que repudia el internacionalismo y pondera la soberanía les permitían demandar un apoyo que, según ellos, "nunca debe degenerar en una intervención militar extranjera: la liberación de Guatemala es el trabajo del pueblo de Guatemala" (FOIA/ERR, Guatemalan Delegation 1954: 2). Si bien el interés principal de la CIA era enfocar la atención en el país centroamericano, especularon que la reunión podría activar grupos anticomunistas privados en el hemisferio, lo cual: "sería útil y contribuiría al logro de los objetivos de la política exterior de los E.U. en el área" (FOIA/ERR, Anti-Communism in Mexico-Specific-Frente Popular Anti-Comunista de México 1954: 1-3).

\section{ANTICOMUNISMO GUATEMALTECO}

\section{EN EL I CONGRESO CONTRA LA INTERVENCIÓN}

SOVIÉTICA EN AMÉRICA LATINA

El 15 de mayo de 1954 atracó en Puerto Barrios el buque sueco Alfhem, para desembarcar pertrechos de segunda mano comprados a Checoslovaquia por el gobierno arbencista, lo que ocasionó una crisis política y diplomática (Perutka 2013: 59-76). Estados Unidos puntualizó el peligro que estas armas significaban para la seguridad del Continente. En medio de estos conflictos, el I Congreso Continental Anticomunista o I Congreso Contra la Intervención Soviética en América Latina se realizó del 27 al 30 de mayo de 1954 en el Teatro Cervantes de la Ciudad de México, presidido por el almirante Carlos Penna Boto, presidente de la Cruzada Brasileña Anticomunista, y Prieto Laurens como secretario general, ambos dieron la bienvenida a las 14 delegaciones representantes de distintos países de la región. Existieron prohibiciones por parte de la CIA para que asistieran organismos o personas de origen estadounidense, lo que no detuvo a los miembros de la Legión Americana, conformada por militares 
veteranos, para presentarse en algunas actividades programadas como la colocación de una ofrenda floral en el Ángel de la Independencia (El Informador 1954a: 1 y 3). Pese a que una de las intenciones del Centro de Operaciones KuGOWN era la asistencia de quienes se destacaran por su anticomunismo y que contaran con prestigio político e intelectual para dar respaldo al evento, solo un pequeño grupo cumplió con las expectativas.

La delegación guatemalteca, aun con la desaprobación de la Agencia, fue desmesuradamente numerosa (FOIA/ERR, Project PBSUCCESS, RYBAT/ PBSUCCESS 1954: 175), conformada aproximadamente por cuarenta miembros, ${ }^{4}$ la mayoría colaboradores directos del plan PBSUCCESS que se encontraban en México, Honduras y El Salvador. Fue encabezada por Luis Arenas, acompañado por Carlos Salazar Gatica, ${ }^{5}$ y el portavoz del CEGAM, Calderón Salazar, como representante oficial de la delegación (FOIA/ERR, Letter for José Calderón Salazar, KuGOwn 1954: 1-3). Su primera ponencia, a cargo de León de Gandarias Iriarte, ${ }^{6}$ causó polémica y varias horas de

4 Los delegados guatemaltecos fueron: Alberto Raúl Arriola Ligorría, Alejandro Baltazar Maldonado Aguirre, Alfredo Eduardo Lürssen Barrios, Arnoldo Orantes Martínez, Buenaventura Echeverría Soto, Buenaventura Vásquez, Carlos Eduardo Taracena de la Cerda, Carlos F. Mendizábal, Carlos Salazar Gatica, Cosme Viscovich Palomo, Darío Soto Molina, Darío Soto Montenegro, Domitila Mérida Palacios, Elena Ramírez Vda. de García, Enrique Coronado Hernández, Evangelina Cuéllar Lemus, Federico Paíz Herrera, Guillermo Putzeys Rojas, Horacio de Córdoba y Monzón, Jorge Palacios Castillo, José Calderón Salazar, José Luis Arenas Barrera, León de Gandarias Iriarte, Lionel Sisniega Otero, Luis Coronado Lira, Luis David Arturo Eskenasy Cruz, Manuel Ángel Orellana Portillo, Manuel Matheu Piloña, Mario López Villatoro, Miguel Antonio Palacios, Olga Marina Orellana, Olivia N. de Archila, Oswaldo Ubico Lemus, Ramiro Aguilar González, Raúl Secaira, René Barrientos D., Ricardo Lara Gálvez, Roberto Gómez de León, Rodrigo Martínez Acosta, Rubén Darío Villatoro Barrios.

5 Salazar Gatica era el abogado de la UFCO y su filial, la International Railways of Central America y socio de Coronado Lira. Su padre era el abogado Carlos Salazar Argumedo, canciller del general Jorge Ubico Castañeda, carrera que heredó a su hijo. En 1953, juntó al expresidente Federico Ponce Vaides, intentó organizar un grupo que penetraría a Guatemala desde la frontera sur mexicana (AHGE-AHD, supuesto movimiento armado contra el gobierno de Guatemala 1953, III-1488-2, fs. 47-53).

6 El editor y escritor León de Gandarias Iriarte fue hijo del escultor y pintor catalán Justo de Gandarias y Planzón, quien llegó a Guatemala a principios del siglo xx para reorganizar la Academia de Bellas Artes. Léon fue redactor en el diario del FND en las elecciones de 1944. 
debate al proponer una declaración de principios anticomunistas y utilizar el concepto de Democracia contrario a lo definido por el Congreso, el cual hablaba de Democracia cristiana sin discriminar a otros credos.

Al clausurar la primera sesión, Calderón Salazar aprovechó para elevar una plegaria, enfatizar su fe católica y poner a su país en la mesa de debate; planteó los objetivos de su lucha que, para ellos, era la liberación de su país encabezados por el coronel Carlos Alberto Castillo Armas. Prieto Laurens pidió a los periodistas presentes que tomaran nota sobre la condición de exiliados de los miembros de esta delegación y puntualizó que eran "los representantes verdaderos de los pueblos, no los representantes de los tiranos" (FOIA/ERR, Preside el Sr. Jorge Prieto Laurens 1954: 39).

Estas palabras dieron pie para que Horacio de Córdoba y Monzón ${ }^{7}$ explicara que la condición en la que vivían en México y Honduras no era por cobardía ni por apátridas, sino porque eran víctimas de la represión del gobierno arbencista y que, al ser detenidos, torturados y defenestrados en su país, luchaban para regresar victoriosos (FOIA/ERR, preside el Sr. Jorge Prieto Laurens 1954: 48). En este sentido, su categoría de desplazados territoriales estimuló sus valores nacionalistas que, en primer lugar, están sólidamente estructurados, como Arturo Taracena señala, en: "un proyecto económico y político oligárquico, de origen colonial, que se ha beneficiado del mantenimiento y recreación de las diferentes etnias" (Taracena 2002: 35). Esta exaltación patriótica es un fenómeno político en el que interactúan tanto el propio Estado y su relación en la construcción, transformación y/o modernización de sí mismo y la nación, como las estrategias utilizadas para conservar el poder o acceder a él. A los participantes del Congreso les permitió identificarse plenamente como una colectividad que disputó la hegemonía política y legitimó su presencia, ideología y objetivos de movilización a través de las múltiples representaciones simbólicas y del proyecto de nación concebido por ellos, el cual

Córdoba y Monzón conducía los radioprogramas: La bora anticomunista de Guatemala y Tribuna de la verdad. En febrero de 1954 aseguró haber sido torturado y arrojado en Chiapas junto a Guillermo Dávila Cordóba, el piloto aviador Gabriel Férnandez, Jorge Palacios y Rúben Darío Villatoro Barrios al ser acusados de participar en un complot antigubernamental (El Heraldo 1954: 1 y 4 ). 
estaba sintetizado en el "Plan de Tegucigalpa" (Comité de Estudiantes Universitarios Anticomunistas [CEUA] 1956), documento difundido desde el 23 de diciembre de 1953. Este era una plataforma del liberacionismo y soporte del movimiento encabezado por Castillo Armas, estructurado como un emotivo documento ideológico con una visión utópica, y a pesar de primar los objetivos y principios básicos en materia económica, política, social e institucional, está lejos de considerarse como parte de una teoría en estas áreas.

El regreso a la patria para estos hombres tenía como fin erradicar al comunismo y, como una más de sus antinomias, asegurar la libertad de opinión política. Así mismo, la refundación del Estado, ${ }^{8}$ a partir del desarrollo económico, contempló impulsar un sistema capitalista moderno, entendiéndolo como la procuración de medidas proteccionistas tanto en la producción agrícola como en la industria extractivista, prácticas que permitirían la entrada de capital extranjero; además de asegurar el derecho y defensa de la propiedad privada.

Es importante mencionar la presencia significativa de mujeres en el Congreso. Ejemplo de ello fue la presentación de Domitila Mérida Palacios, ${ }^{9}$ en la que se destacó la necesidad de emigrar de su país y la forma en que ellas podrían integrarse a la lucha desde el exilio, como "un soldado

8 Si bien, esta idea se fundamenta en el documento mencionado, es decir, el Plan de Tegucigalpa. El esfuerzo del gobierno de Castillo Armas por llevar a cabo dicha refundación se proyectó en la reestitución, reformulación y creación de nuevos códigos y leyes que, desde la creación del Estatuto Político de la Junta de Gobierno (Junta de Gobierno 1954), en agosto de 1954, se confirió un marco legal y político hasta que fue sustituido por una nueva Constitución. Posteriormente, se creó el Consejo Nacional de Planificación Económica que redactó el Plan de Desarrollo Económico de Guatemala, el cual delineó la explotación de recursos naturales, la modernización de la infraestructura, la reestructuración del agro y la regulación de las relaciones laborales (Castillo 1956). Con ello, también se institucionalizó la violencia contra la oposición, basicamente, de cáracter comunista. Esto se proyecta en la publicaciones que se realizaron en los talleres de la Tipografía Nacional y el arduo trabajo de la Secretaría de Divulgación, Cultura y Turismo de la Presidencia de la República.

9 Mérida Palacios intentó formar el Partido Obrero Cristiano junto a Eduardo Arriola Ligoria, Antonio Morán, Óscar Luna Campos, Horacio de Córdoba y Monzón, el cual, finalmente, se conformó como el Comité Obrero Anticomunista (COA) (FOIA/ERR, Miscellaneous Political Activity 1954: 3). 
más al servicio de la Patria" (FOIA/ERR, Preside el Sr. Jorge Prieto Laurens 1954: 274). También enfatizó en la persecución y vigilancia a la que eran sometidas por pertenecer a la oposición, y dejó claro el papel subalterno de las mujeres en esta lucha. Asimismo, insistió en la abnegación que debe caracterizar al anticomunista que se guía por medio de la caridad cristiana.

Durante la segunda sesión, Luis Arenas continuó con las invocaciones religiosas para señalar que el comunismo "lucha fundamentalmente contra dios, niega su realidad y su providencia" (FOIA/ERR, Additional publicity 1954: 3). Después, a manera de protesta, pidió un minuto de silencio y retomó el discurso del arzobispo Mariano Rossell y Arellano -máximo representante de la Iglesia de su país desde 1939-, quien homologó a la lucha anticomunista con una cruzada espiritual, una misión apostólica de dimensión nacional. Esto dio el fundamento religioso para el exterminio del enemigo por medio del sacrificio y el derramamiento de sangre como algo circunstancial. Este pensamiento fue sintetizado a través del lirismo de Calderón Salazar cuando alude al escudo liberacionista: "Y esto no puede llamarse revolución: se llamará Cruzada. Porque el quetzal en vuelo traza el misterioso signo de la cruz en el aire, como el águila, como Moisés orando ante el inacabable fuego de la zarza" (1955: 24-25). La declaratoria acentuó la disociación de los gobiernos revolucionarios con su misión, en cuanto a que su ideal de patria implicó concebir a Guatemala como el pueblo elegido. ${ }^{10}$ Secundó esta postura Alberto Raúl Arriola Ligorría, ${ }^{11}$ quien expresó que el deber del católico en América Latina era aceptar la Doctrina Social Cristiana, sustentada en las encíclicas papales Rerum Novarum ${ }^{12}$

10 La éxegesis de la zarza ardiente frente a Moisés (Éxodo 3: 1-3) tiene diferentes interpretaciones, una de ellas es que simboliza al Pueblo de Dios, lo que implica que jamás será destruido.

11 Arriola Ligorría es hijo de Manuel Ismael Arriola Porres, quien ocupó puestos públicos en los años veinte del siglo XX. Su hermano Jorge Luis fue ministro de Educación, nombrado por la Junta de 1944, donde decretó la autonomía de la Universidad de San Carlos y, durante los gobiernos revolucionarios, ostentó diversos cargos públicos, principalmente en el servicio exterior. Por su parte, su hermano Eduardo trabajó junto a él en el COA.

12 El 15 de mayo de 1891, el Papa León xIII publicó Rerum Novarum. Sobre la situación de los obreros (De las cosas nuevas). Plantea la búsqueda de una justicia social, así 


\section{y Divina Redentora ${ }^{13}$ (FOIA/ERR, Additional Publicity 1954: 1-11). Agregó} que el comunismo desconocía los principios fundamentales de la familia, lo que era un atentado contra esta institución social y los designios divinos que resguardan la catequesis, la edificación del Reino de Dios y la base esencial de su obra. ${ }^{14}$ En concordancia con estos preceptos, el plan de gobierno liberacionista contempló al matrimonio como "la base de la familia" que, junto a la maternidad, deberían tener la protección del Estado.

Como contraste, consideraba que el comunismo elevaba al hombre a semejanza de Dios, su creador, es decir, aludió al epíteto de falsa redención que desencadena "una lucha fríamente calculada y cuidadosamente preparada contra 'todo lo que es divino"' (Pío XI 1937: 73). Esta disertación remite al trabajo en Guatemala del Secretariado Social Rerum Novarum que tuvo como órgano de difusión al semanario Acción Social Cristiana,${ }^{15}$ constituida por laicos aglutinados en Acción Católica y que reprodujo, junto a Verbum ${ }^{16}$ el pensamiento del trabajo pastoral que se

como la defensa de la propiedad privada y el derecho al sindicalismo. Es la carta fundacional de la Democracia Cristiana.

13 El 19 de marzo de 1937, el Papa Pío xI publicó Divini Redemptoris. Sobre el comunismo ateo. En ella alude al papel de la Iglesia frente al marxismo, que replanteó la Doctrina social de la Iglesia.

${ }^{14}$ Esta idea se entrecruza con el pensamiento del Papa León XIII, quien señaló que "los socialistas, descuidada la providencia de los padres", introducen en su lucha la del Estado, obran "contra la justicia natural", y "disuelven la trabazón del hogar doméstico" (189: 7).

15 Ante las libertades del régimen que se establecía en 1944, el Secretariado Social Rerum Novarum fue fundado por el padre jesuita Isidro Iriarte Aguirrezábal junto a varios jóvenes católicos de las elites familiares criollas como los Urruela y los Aycinema Salazar, además de otros sectores vinculados a estas como Alaide Foppa Falla y Antonio du Teil. Este último alternó el cargo de director de su órgano de difusión Acción Social Cristiana con Juan Alberto Rosales Flores. En 1945, con la salida del padre Iriarte hacia El Salvador, su compañero de hábito, el padre Agustín Bariaín, asumió brevemente sus responsabilidades en el grupo, para después tomar dicha dirección otro jesuita, el padre Carmelo Sáenz de Santamaría, todos de origen vasco.

16 Verbum fue el semanario oficial del Arzobispado. Publicó su primer número el 8 de marzo de 1942, bajo la dirección del padre jesuita de origen vasco, Ángel Arín Omazábal y Caballero. Arín tenía una sólida formación intelectual y su teología se basaba en el tomismo, predicaba en la catedral guatemalteca junto al padre Iriarte, quienes tuvieron problemas con el gobierno por el cáracter político de sus sermones. Junto 
enfocó, entre otras cosas, en luchar contra el comunismo y en atender el precepto papal de recristianización.

Para Pío XI, los miembros de Acción Católica eran soldados que "serán los primeros e inmediatos apóstoles de sus compañeros de trabajo y los preciosos auxiliares del sacerdote" (1937: 86). Esto fue una de las preocupaciones del arzobispo Rossell quien, desde el 22 de febrero de 1945, estableció su postura a través de la carta pastoral colectiva del Episcopado de la Provincia Eclesiástica de Guatemala titulada Sobre la amenaza comunista en nuestra patria (1945), circunscrita a las encíclicas mencionadas. Además, dicha comunicación sataniza al comunismo, lo caracterizó como apóstata, idólatra, hereje e inmoral, es decir, como parte integral y perpetua del enfrentamiento entre el bien y el mal. Perfiló, además, el deber de los feligreses y llamó a cristianizar la vida económica y a la caridad cristiana. Interpretó que este trabajo sería posible a través de la justicia social, cuya atribución es "exigir de los individuos cuanto es necesario al bien común" (1954: 3) y que combatiría la miseria del obrero y el campesino para alejarlos de la tentación del comunismo, planteamientos que posteriormente serán retomados en el Plan de Tegucigalpa.

Al principio de los gobiernos revolucionarios, la relación del arzobispo con estos fue moderada, posteriormente radicalizó su oposición al ser suprimidas algunas libertades al clero y al ser acusado, junto a la Iglesia guatemalteca, de participar en asuntos de carácter político y su relación con el falangismo. El 4 de abril de 1954 publicó la carta pastoral Sobre los avances del comunismo, donde exhortó a todo católico a levantarse contra este enemigo "de Dios y de la Patria" (Rosell 1954: 2). Es esta atmósfera la que permeó en los delegados guatemaltecos ${ }^{17}$ y les permitió,

a Acción Social Cristiana fue un medio que ejerció presión política a los gobiernos revolucionarios.

17 Calderón Salazar recopiló una serie de textos que fueron leídos al aire en la Radio Nacional de Guatemala para celebrar el primer año del triunfo liberacionista. En ellos se expresa profundamente el ideario de los anticomunistas guatemaltecos aglutinados en la Oposición Organizada, muchos de ellos delegados en el evento que aludimos. Publicado bajo el título Letras de liberación, su contenido posee un fuerte sesgo católico basado en el Viejo Testamento, el cual nos permite comprender la importancia que tuvo la Iglesia y su doctrina para dotar de símbolos a su lucha (Calderón 1955). 
en primer lugar, articular su militancia y, como praxis de esta, paradójicamente priorizar el uso de la violencia como forma de lucha. Al mismo tiempo, fueron capaces de construir el arquetipo del buen cristiano, en cuanto proyectaron la construcción de un nuevo Estado y la refundación de la nación.

Estas ideas tuvieron eco en el falangismo en Guatemala ya que, desde el reconocimiento al gobierno del general Francisco Franco por el general Ubico en 1936, se permitió abiertamente su difusión, básicamente a través de la revista Amanecer ${ }^{18}$ y el apoyo del servicio exterior. ${ }^{19}$ Durante el periodo ubiquista, el 12 de diciembre de 1937 se constituyó la Falange guatemalteca, fomentada por el representante diplomático español Rafael Triana y Blasco y en la jefatura su cónsul, Enrique García-Rendueles (Delgado 1948: 78). Posteriormente, pese al desconocimiento del gobierno franquista por parte de la junta revolucionaria de 1944, dicha difusión se dio a través de los medios de comunicación de la Iglesia católica y de las redes familiares que establecieron con la elite local, vinculados también a la acción social de los laicos. ${ }^{20}$

18 La Revista Amanecer era el Órgano de la Falange Española Tradicionalista de las Juntas de Ofensiva Nacional Sindicalista, dirigida por el delegado de Prensa y Propaganda asignado por el Servicio Exterior de la Falange en Guatemala, Herminio Rodríguez Quijano. Además de aglutinar a los españoles que compartían la ideología y trazar las directrices de su militancia, el Servicio Exterior buscó establecer vínculos con organizaciones anticomunistas y fascistas en los territorios en el que pudieran tener libertad de movimiento.

19 El Consúl repúblicano español en Guatemala era Francisco López Escobar. Al terminar la guerra, hubo una reestructuración y depuración de los diplomáticos, por lo que fue destituido, se quedó en el país y buscó ganarse la confianza del gobierno franquista, lo que consiguió hasta el 8 de enero de 1939 cuando fue admitido para prestar servicio. En tanto que el 24 de mayo de 1937 se nombró como ministro plenipotenciario y primer consúl a Rafael Triana y Blasco hasta el 21 de diciembre de 1940, cuando fue sustituido por el coronel Antonio Sanz Agero. El 29 de abril de 1941, el Ministerio de Asuntos Exteriores franquista propuso a Jorge Ubico para nombrarlo Caballero del Collar de la Orden de Isabel la Católica.

20 Un ejemplo de ello fue el matrimonio entre el delegado de Trabajo de la Falange, el ingeniero y poeta José María Sagone Ibañez y un miembro de las familias más poderosas en el país, Rosa Aycinema Salazar. El abogado Pedro Aycinema, hermano de Rosa, fue fundador del Secretariado Social Rerum Novarum. 
En lo político, aludieron a la x Reunión de la Organización de Estados Americanos (OEA), realizada del 1 al 28 de marzo de 1954 en Caracas, Venezuela. Básicamente se referían a la resolución XCIII, ${ }^{21}$ que, si bien tenía un corte anticomunista, aseguraron que eran posturas diplomáticas. A través de un comité ejecutivo del Congreso contra la Intervención Soviética en América Latina plantearon un llamado a este organismo internacional para que se considerara su caso y se tomaran medidas que detuvieran el intervencionismo soviético, para posteriormente llevar el caso al Consejo de Seguridad de la Organización de Naciones Unidas, pues su avance constituía "una amenaza a la soberanía e independencia política de los Estados americanos" (Otarola 1956: 175) y ponía en peligro la paz.

La aportación de los guatemaltecos que participaron en la reunión rápidamente tomó relevancia en los titulares de los diarios y algunos de ellos fueron entrevistados para informar sobre el evento (El Informador 1954b: 1). Asimismo, sus planteamientos estuvieron circunscritos al carácter represor y criminal del gobierno de Árbenz. Como se puede apreciar en las palabras de Córdoba y Monzón, que aseguró que la representación obrera de su país quedaba vacía en el Congreso al ser asesinado el secretario general del COA, el mecánico Óscar Luna Campos (FOIA/ERR: Preside el Sr. Jorge Prieto Laurens 1954: 132-134). ${ }^{22}$ Los asistentes, de inmediato, lo adoptaron como mártir y ofrecieron al siguiente día un pendón floral a cargo de Arenas Barrera. Para ellos era trascendental instrumentalizar a sus

21 La resolución se titula "Declaración de Solidaridad para la Preservación de la Integridad Política de los Estados Americanos Contra la Intervención del Comunismo Internacional" y fue fomentada por Estados Unidos a través de su secretario de Estado, John Foster Dulles. Fue aceptada con 17 votos a favor de los 21 países representados en la reunión, en tanto que México y Argentina se abstuvieron y el único voto en contra fue emitido por el canciller guatemalteco Guillermo Toriello Garrido. Cabe resaltar que el presidente costarricense, José Figueres Ferrer, no envío representación a la reunión al prever los resultados de ésta.

22 Óscar Luna Campos, de 32 años de edad, militaba en el PuA, en la sección del coA. Al ser electo delegado en el Congreso en México Horacio Córdoba, Óscar Luna tomó su lugar en La bora anticomunista. El 2 de mayo de 1954, su cuerpo fue encontrado en el Lago Atitlán con visibles marcas de tortura. Su esposa, María Trinidad Cruz, fue la única mujer que se integró en las filas del ejército liberacionista (FOIA/ERR, Report re Guatemala 1954 coup 1954: 2). 
muertos como parte de su doctrina, "rendir culto a los héroes, a fortalecer su memoria y a exponerlos como paradigmas del pueblo" (Calderón 1955: 14). Este tipo de acciones eran la expresión de su nacionalismo moldeado con valores católicos, dotaban de símbolos a su misión y exponían con claridad la idea de sacrificio que conllevaba esta, como lo exaltó Arenas Barrera: "Así estamos lo guatemaltecos dispuestos a morir de pie" (FOIA/ERR, Preside el Sr. Jorge Prieto Laurens 1954: 201). En este sentido, Coronado Lira, al referirse a su bandera, expresó: "Tendrá un asta hecha con todos los brazos de los guatemaltecos, con nuestra sangre y con nuestra vida, con nuestro sacrificio y, si Dios quiere, con nuestra misma muerte" (Calderón 1955: 33). Lo anterior, como recurso expiatorio, da cumplimiento al precepto de la caridad cristiana retomada por Pío XI, quien señaló que era necesario "olvidarse de sí mismo, por el amor al prójimo" (1939: 81), por lo que el enfrentamiento contra sus adversarios se interpretó como el flagelo corporal y espiritual inevitable, que incluso podría llegar a la inmolación para redimirse. Estas intervenciones enmarcaron la ponencia principal de la delegación, titulada: "El caso de Guatemala", que:

no constituye un arma de combate contra el gobierno de Guatemala. Es un Libro Blanco de la Oposición Guatemalteca Organizada, que ha de servir de testimonio histórico, de que su lucha contra el comunismo realmente es dirigida para liberar a la Patria de la subyugación soviética y no una simple bandera para derrocar al gobierno (Secretaría de Divulgación, Cultura y Turismo de la Presidencia de la República ([SDCyT] 1956: 351).

El texto incluía pruebas como fotografías, recortes de periódicos y diversos documentos que pretendían demostrar irrefutablemente la penetración soviética en su país y, por ello, conferir legitimidad y legalidad a su lucha. Dicho documento fue aceptado para que se enviase a la Reunión de Consulta de Cancilleres que estaba programada para celebrarse en Washington el 7 de julio próximo, donde se trataría este tema. ${ }^{23}$

23 Las Reuniones de Consulta de cancilleres se realizan para tratar asuntos considerados de carácter urgente y de interés común para los miembros de la OEA. Ante la situación que enfrentaba Guatemala, el Consejo Permanente decidió cancelar la reunión, por lo que no se concretó. 
Además, incluía una recriminación a los gobiernos de Arévalo y Árbenz por romper los compromisos avalados en las Conferencias Interamericanas de 1947 en Río de Janeiro, Brasil, donde se estableció el "Tratado Interamericano de Asistencia Recíproca (TIAR)" y en Bogotá, Colombia, en 1948, donde se creó propiamente la OEA y se firmó el "Tratado Americano de Soluciones Pacíficas" y la "Declaración Americana de Derechos y Deberes del Hombre". Esto implicaba la imposibilidad de intervención o participación directa en Guatemala por otras naciones miembros de la OEA. El artículo 3, párrafo 1, del TIAR establece que ante cualquier: "ataque armado por parte de cualquier Estado contra un Estado Americano, será considerado como un ataque contra todos los Estados Americanos" (1947). De esta manera, los miembros del organismo tenían el compromiso de involucrarse en su defensa. Ante ello, su urgencia de enviar la información recabada a la Reunión de Consulta de cancilleres era para que validaran el artículo 6 del Tratado, en el que se expresa que, si este ataque no es armado, pero pone en riesgo la paz del continente, el Órgano de Consulta decidirá las medidas precautorias o de salvaguardia, en las que se involucra a otras naciones americanas. Les era imperante demostrar que el riesgo no recaía únicamente en la injerencia soviética hacia América ni en la ideología del gobierno arbencista, si no que pretendían demostrar la transnacionalización del conflicto, al fomentar:

revueltas armadas y ayudando a estas con dinero, armas, pertrechos, gente, etc., ya agitando en terrenos laborales o agrícolas, alentando huelgas o financiándolas, ya introduciendo propaganda marxista subrepticiamente, en forma de libros, folletos, gráficos y hasta enviando técnicos en las labores del proselitismo comunista (FOIA/ERR: Additional publicity 1954: 8).

El material fue presentado a una comisión para que se estudiara. Si bien las delegaciones presentes en el Congreso tomaron la decisión de emprender acciones contra el peligro que representaba la presencia soviética en este país - que ponían en riesgo a todo el continente-, su principal interés era incidir en las decisiones de la Reunión de Consulta para que les permitiera la participación de sus Estados en los planes de invasión a territorio guatemalteco. 
También buscaron explotar la acusación contra los gobiernos revolucionarios de cometer delitos flagrantes contra los derechos humanos (El Informador 1954c: 1 y 3). Los testimonios sobre vejaciones, torturas, privación de libertad, persecución, censura y exclusión política y civil a las que fueron sometidos algunos exiliados estaban enfocados en exponer que estos abusos trasgredían la Declaración Americana de los Derechos y Deberes del Hombre (1948). Si bien las sanciones al Estado guatemalteco no eran de orden administrativo o jurídico, podrían impulsarse desde el orden moral.

Los reportes de la CIA, firmados por Boyd I. Rolender, señalan su satisfacción por el rechazo generado contra el gobierno arbencista y por la petición de prestar atención al caso guatemalteco hecho a la OEA (FOIA/ ERR, Report on el Primer Congreso Contra la Intervención Soviética Part I: 1-3). Básicamente, exigían apegarse al artículo 1, que refiere a la naturaleza de su organización y dicta que se debe "lograr un orden de paz y justicia, fomentar su solidaridad, robustecer su colaboración y defender su soberanía, su integridad territorial y su independencia" (1948). Asimismo, acogerse a los artículos 4 y 40 , concernientes a los miembros y a los tratados comerciales que sugerían la ilegalidad de las compras de pertrechos checos por parte del gobierno de Árbenz. Ante todo, la Agencia estadounidense juzgó al encuentro como un éxito de la operación PBSUCCESS (FOIA) ERR, Cable to director, Lincoln from [deleted] re Guatemala 1954 coup 1954: 1-2), ya que la operación asignada a sus agentes Boyd I. Rolender y Joel E. Nebecker fue "muy exitosa y una contribución definitiva al proyecto" (FOIA/ERR, Project PBSUCCESS, RYBAT/PBSUCCESS 1954: 175) y podría ser de utilidad en el futuro.

Pese al evidente flujo de recursos y apoyo logístico por parte de los Estados Unidos a través de su Agencia de Inteligencia, los organizadores jamás aceptaron esta intromisión, inclusive se empecinaron en negarlo para proyectar una perspectiva centroamericana del triunfo liberacionista. Ello se aprecia en las memorias de Prieto Laurens, quien afirma que: "lo hicieron con elementos propios y con la valiosa ayuda de los pueblos hermanos de El Salvador, Honduras y Nicaragua" (1968: 347). Además, aseguró que los Estados Unidos "con su política vacilante e indefinida, se 
limitaron a contemplar los acontecimientos" (1968: 347). Con tal afirmación, Prieto Laurens fingió ignorar las maniobras políticas que se realizaron en los organismos internacionales y el financiamiento recibido por los liberacionistas y los organizadores del Congreso por parte de la CIA. En contraposición, las evaluaciones de Rolender señalan que en la obsesión de Laurens por señalar la influencia comunista en el gobierno mexicano, no solo se ridiculizó él, sino al Congreso, pues fueron vituperados en los diarios nacionales que destacaron los imprevistos y el rechazo que ocasionó la reunión. Como contraste, a la Agencia le agradaron los resultados del encuentro, reconocieron que no fue una operación específica, "por el contrario, es una operación integral con muchas facetas, las cuales deben mantenerse bajo un estricto control para garantizar un producto final completamente aceptable" (FOIA/ERR: Report on El Primer Congreso Contra la Intervención Soviética en América Latina- Parte II 1954: 2-3).

Por su parte, los organizadores del evento continuaron con sus planes. El objetivo subsecuente era proyectar internacionalmente la imagen caudillista de Castillo Armas, sobre todo en los Estados Unidos. Prueba de ello es una carta escrita por el secretario de relaciones públicas del FPAM, Florencio Ávila, conservada por la agencia estadounidense y dirigida a Castillo. Indica que buscara editoriales a su favor y usara sus contactos en la televisión y los medios escritos de aquel país, y asegura que presionarían al Departamento de Estado, a través de sus contactos como los senadores Alexander Wiley, Joseph McCarthy y Willian Fife Kwonland, para que le brinden apoyo. ${ }^{24} \mathrm{~A}$ la par del trabajo que se realizó a través del aparato mediático de la misma CIA, en el cual destaca la actividad del ex oficial de inteligencia y periodista estadounidense Jules Dubois, quien fue instructor de Castillo Armas en Fort Leavenworth (Bozza 2019: 138-147). Los delegados guatemaltecos, en su mayoría militantes de la Oposición

24 Sus contactos eran Walter Winchel, Westbrook Pegler, Drew Pearson, Howard Rushmore, la Cadena Hearst, propiedad de William Randolph Hearst; Henry Robinson Luce, dueño de las revistas Time y Life; y por la fundación Facts Forum del magnate petrolero Haroldson Lafayette Hunt (FOIA/ERR, Note to P.D. From Pancho 1954: 1-5). 
Organizada y activos en las estructuras del Ejército de Liberación, regresaron a sus lugares asignados en México, Honduras, El Salvador y Guatemala para continuar con los planes para penetrar a territorio guatemalteco.

Tres semanas después de concluir el Congreso, el 18 de junio de 1954, Castillo Armas inició una invasión al frente del Ejército de Liberación Nacional que cruzó la frontera desde Honduras. Después de 11 días de intervención Árbenz Guzmán dimitió a la presidencia, sucediéndose en el poder político diferentes juntas militares, hasta que el líder liberacionista logró el control. Algunos asistentes al Congreso fueron parte del nuevo gabinete, como Salazar Gatica, nombrado ministro de Relaciones Exteriores, y Coronado Lira, como secretario particular, mientras otros se mantuvieron en la órbita de la nueva administración permitiéndoles la hegemonía política. ${ }^{25}$

Su participación en el Congreso les aseguró la libertad para desmantelar el régimen democrático anterior y dar paso a la transformación de las relaciones de poder. Esto derivó en la persecución, hostigamiento, desarticulación y aniquilamiento de cualquier persona, grupo o expresión que significara la continuidad del proyecto arbencista o de las ideas marxistas. Dicho proceso fortaleció la autoridad de su jefe máximo, que posteriormente legitimó su presidencia a través de un plebiscito realizado el 10 de octubre de 1954.

25 Orellana Portillo, Lopéz Villatoro, Calderón Salazar, Arriola Ligorría y Arenas Barrera fueron diputados en la Asamblea Nacional Constituyente; Sisniega Otero, subsecretario de Gobernación; Orantes Martínez, agregado de prensa en la Embajada en México; Lara Gálvez, 1er juez de Paz; Gómez de León, 1er secretario en la Embajada en República Dominicana; Taracena de la Cerda, secretario particular de Castillo Armas; Paíz Herrera, subsecretario de Educación Pública; Villatoro Barrios, presidente del Comité Ejecutivo del Comité Nacional de Reorganización Sindical; y De Gandarias Iriarte, jefe de redacción del Ministerio de Gobernación. Los demás se afiliaron al Movimiento Democrático Nacionalista, partido creado por orden de Castillo Armas. En tanto que el capitán Secaira y Viscovich Palomo murieron durante la invasión liberacionista. 
En el mes de julio, el ministro de Relaciones Exteriores, Salazar Gatica, junto con el secretario particular, Coronado Lira, el subsecretario de Gobernación, Sisniega Otero, el secretario privado de la Junta de Gobierno, Óscar Cobar Castillo y Eduardo Taracena de la Cerda, recibieron a la comitiva del Congreso integrada por Prieto Laurens, los periodistas Mariano Quirós González, representante de la Juventud Anticomunista de Costa Rica, y Antonio Porto Sobrinho, representante de la Juventudes Democráticas de Brasil, quienes mostraron su respaldo al nuevo régimen y avalaron el informe presentado en México. Este escrito originalmente era la ponencia: "El caso de Guatemala", texto que fue editado y ampliado con diversos documentos incautados por la CIA durante los primeros días de la invasión. Se publicó en diciembre de 1954 con el título: El libro negro del comunismo en Guatemala, bajo el sello editorial de la Comisión Permanente del Primer Congreso contra la Intervención Soviética en América Latina. No obstante, los documentos internos de la Agencia me permiten asegurar que fue una publicación de la Central Intelligence Agency-United States Information Service (CIA-USIS) (FOIA/ERR, Use of Documents Collected in Guatemala by United States Team after Establishment of Castillo Armas Government 1954: 2), quien conformó el corpus de obras auspiciadas por la Agencia para que se difundiera la idea de la intervención soviética y la implementación del comunismo. Por otro lado, la intención de que el texto original influyera en la decisión de la Reunión de Consulta de Cancilleres se interrumpió al cancelarse dicho encuentro ante la invasión liberacionista. No obstante, el titubeante llamado a detener "toda actividad que pueda provocar un derramamiento de sangre" (Consejo de Seguridad de la Organización de Naciones Unidas. Resolución 104, 1954), que dio como respuesta el Consejo de Seguridad de la onu a la denuncia del gobierno guatemalteco, complació a los delegados anticomunistas, pues les aseguró un campo de acción para lograr sus objetivos.

Aunque no existe un nombre o nombres vinculados a la autoría del texto, algunos investigadores que han estudiado e interpretado el contenido del libro se lo han atribuido erróneamente a la Comisión Permanente 
del Congreso (Grijalva 2019: 1, 4 y 7). Por su parte, Enrique Camacho Navarro, al hacer un análisis de las representaciones en el texto, señala que es una autoría corporativa y lo ubica como un instrumento que permite la construcción negativa de la imagen de los comunistas guatemaltecos. Aborda, también, diferentes tópicos que se entrelazan con el devenir latinoamericano y destaca que "Este material es muy elocuente desde su propia portada" (Camacho 2006: 83-119). La cual es sumamente estridente, presenta en el centro un quetzal con las alas extendidas en color verde, el ave desgarra una tela roja en la que hay un boceto de la hoz y el martillo; en la parte superior, en letras negras, se lee el título del libro y, en la parte inferior, el nombre de los editores con su domicilio. El texto, que irónicamente se mostró como El Libro Blanco de la Oposición Guatemalteca Organizada, se presenta como corolario del trabajo de propaganda realizado por el Centro de operaciones KugOwn, la Secretaría General de la Comisión Permanente del Congreso, los delegados guatemaltecos y las autoridades del nuevo régimen, por lo que se difundió ampliamente.

La agencia estadounidense enfocó su interés en otras tareas, básicamente en sostener al nuevo régimen y mostrar que se había actuado correctamente al deponer un gobierno influenciado por la doctrina comunista. En tanto que el uso de la propaganda, además del intenso trabajo internacional en Guatemala, se llevó a cabo a través de la Secretaría de Divulgación, Cultura y Turismo de la Presidencia de la República, la cual publicó diversidad de textos que ponderaban la nueva administración, para proyectar la invasión como una gesta patriótica y ensalzar la figura del líder liberacionista.

Estas acciones ayudaron a establecer una serie de representaciones sociales, tanto de sí mismos como de sus adversarios, expresadas a través del discurso codificado que ordenó su estructura ideológica y articuló a la militancia y su acción social. También permitió asegurar su dirección como grupo y conservar el poder político que expoliaron a su enemigo, constituido en la justificación de su lucha y que, ahora como grupo subordinado, requería de un nuevo proceso de domesticación. Por su parte, la Comisión Permanente del Congreso continuó con sus compromisos adoptados encaminados a la organización de la segunda reunión antico- 
munista latinoamericana, proyectada a realizarse en Río de Janeiro, Brasil, en $1955,{ }^{26}$ con toda la fuerza simbólica, moral y política que significó el triunfo de los liberacionistas sobre Árbenz Guzmán.

\section{CONCLUSIONES}

Los resultados del Congreso han quedado en entredicho. Santiago Jiménez, al evaluar algunos reportes de la agencia, concluye que por los errores de la organización y el desarrollo del evento lo hacen lucir como un desastre (2017). Sin embargo, difiero de ello, pues se cumplió en forma cabal el objetivo del centro de operaciones KuwONG, enfocado en presentar el caso guatemalteco y, como parte integral del plan PBSUCCESS, asegurar el respaldo al golpe de Estado que estaba planeado realizarse en días próximos, impulsar el hostigamiento hacía sus enemigos y menguar las denuncias de la intervención financiada y asesorada por la CIA.

Dentro de los objetivos logrados se incitó la interacción entre los grupos y personajes participantes, quienes en su mayoría mostraron una simpatía por las políticas de Estados Unidos hacia América Latina, hombres que, en algunos casos, eran o posteriormente fueron funcionarios públicos en sus respectivos países. El impacto del trabajo realizado tomó notoriedad con el tiempo, se aseguró, en primer lugar, la condena al gobierno de Árbenz y la aceptación general de la invasión encabezada por Castillo Armas y su posterior gobierno, concibieron su triunfo como continental y una derrota del comunismo de la que participaron conjuntamente. En segundo lugar, la labor de guerra sicológica y propagandística continuó a través de las colaboraciones de los miembros de la naciente red anticomunista, reflejada en múltiples editoriales periodísticas y publicaciones bajo

26 Además de la conferencia realizada en Río de Janeiro, Brasil, del 22 al 26 de agosto de 1955, se realizaron dos más, una en Lima, Perú, del 10 al 14 de abril de 1957 y la última en Antigua, Guatemala, del 12 al 15 de octubre de 1958. Después de estas reuniones continentales, se decidió organizar congresos regionales, en el caso centroamericano se llevaron a cabo, primero en San José de Costa Rica, del 10 al 13 de enero de 1959, mientras el segundo, en Managua, Nicaragua, del 24 al 27 de marzo de 1960 (Vázquez 2020: 1-28). 
el auspicio de la organización latinoamericana o la misma CIA. Estos textos se posicionaron como artefactos que moldearon el paradigma e ideario filosófico operativo. Finalmente, esta militancia fundamentó la construcción del nuevo Estado en sus preceptos doctrinarios cimentados en las encíclicas papales mencionadas y que impidieron laicidad en su proyecto de nación, delineado por los principios de "Dios, Patria y Libertad".

Aunque el estudio del contexto en que se desarrolló este Congreso, es decir, el derrocamiento de Árbenz y el estrepitoso ascenso de Castillo Armas a la presidencia, ha sido ampliamente estudiado, estas redes transnacionales y la participación de su militancia se han abordado escasamente. Los discursos que se formularon establecieron representaciones sociales y códigos de comunicación simbólicos que organizaron la movilización social y la articulación de los grupos y de sus militantes. Esto les permitió interconectarse y transferir conocimientos que, al ensanchar su campo de acción, tuvieron injerencia directa en los efectos continentales desde la actividad colectiva regional. En Guatemala, esta militancia no solo ostentó el poder político, sino que tuvieron incidencia en la configuración del nuevo marco institucional. Además de ocupar curules en el Congreso de la República, participaron en la Asamblea Constituyente de 1956, o a nivel regional, penetraron organismos como la misma ODECA y, como se mencionó anteriormente, atrajeron el conflicto ideológico mundial al territorio latinoamericano con características propias. Aunque esta reunión y las que la prosiguieron se desarrollaron con anterioridad a la ola de luchas armadas en América Latina, durante estos años se conformaron los conceptos, axiomas y fundamentos teóricos que dieron paso a la estructuración de un Estado contrainsurgente y a la proliferación de los llamados escuadrones de la muerte, en muchos casos, erigidos por esta militancia.

Para finalizar, al estudiar a los grupos hegemónicos, su acción colectiva y su función como productor de nuevos sentidos, es posible observar las pautas de continuidad de estos hechos sociales en la actualidad. En Guatemala se eligió como presidente a Alejandro Giammattei Falla, vinculado a los cuerpos ilegales y aparatos clandestinos de seguridad, antes llamados: escuadrones de la muerte. Además de arroparse bajo el sector 
empresarial y miembros de la institución castrense para revivir la pena de muerte y resignificar la tipificación de terrorismo, fortalece el proceso de derechización de Latinoamérica, encabezado por el gobierno extremista de Jair Messias Bolsonaro, en Brasil; las manifestaciones populares contra la administración de Sebastian Piñera Echenique, en Chile; el golpe de Estado contra Evo Morales Ayma, en Bolivia y, aún más reciente, el rechazo popular a las medidas sanitarias por la pandemia mundial al vincularlas con acciones comunistas. Estos ejemplos muestran la continuidad del discurso en contra del comunismo en los gobiernos de América latina, sustentados por el miedo y el exacerbado fanatismo religioso.

\section{BibLIOGRAFÍA}

ACuÑa OrTEGA, Víctor HugO. "Centroamérica en las globalizaciones (siglos XVI-XXI)". Anuario de Estudios Centroamericanos 41 (2015): 13-27. Bohoslavsky, ERnesto y Magdalena Broquetas. "Los Congresos anticomunistas de América latina (1954-1958): redes, sentidos y tensiones en la primera Guerra Fría". III Coloquio "Pensar las derechas en América latina en el siglo XX”. Brasil: Universidad Federal de Minas Gerais, 2018. BOzZA, JuAn AlBERTO. "Las espadas mediáticas de la derecha. La Sociedad Interamericana de Prensa en la Guerra Fría Latinoamericana". Almanaque Histórico Latinoamericano 23 (2019): 137-162.

CALDERÓN SALAZAR, José. Letras de Liberación. Guatemala: Tipografía Nacional, 1955.

CAmacho NaVARro, EnRique (coord.). "Imágenes y letras. El poder de las representaciones en la lucha política en Centroamérica y el Caribe". El rebelde contemporáneo en el Circuncaribe. Imágenes y representaciones. México: UnAM-CIALC/Edĕre, 2006: 83-119.

CAÑón, Julio LISANDRO. "La Confederación Interamericana de Defensa del Continente (CIDC)". Rúbrica Contemporánea 12.6 (2017): 79-99.

Casals, MarCelo. "Against a Continental Threat: Transnational Anti-Communist Networks of the Chilean Right Wing in the 1950s". Journal of Latin American Studies (2019): 1-25. 
Castillo Armas, Carlos. Plan de Desarrollo Económico de Guatemala, 1955-1960. Guatemala: Tipografía Nacional, 1956.

Comité De Estudiantes Universitarios Anticomunistas. Plan de Tegucigalpa. Guatemala: CEUA, 1956.

CONFERENCIA InTERAMERICANA PARA EL MANTENIMIENTO dE la PAZ Y la SEGURIDAD DEL CONTINENTE. Tratado Interamericano de Asistencia Recíproca. Río de Janeiro, 1947.

Consejo de Seguridad de la ORGanización de NaCiones Unidas. "Resolución 104”. 1954.

Coy Moulton, Aaron. Guatemalan Exiles, Caribbean Basin Dictators, Operation PBFORTUNE, and the Transnational Counter-Revolution against the Guatemalan Revolution, 1944-1952. Universidad de Arkansas, 2016.

CUllather, NiCHOlLAS. Operation PBSUCCESS: The United States and Guatemala 1952-1954. Central Intelligence Agency, 1994.

DEPARTAMENT OF STATE. Foreign Relations of the United States, 1952-1954, Guatemala-United States, 2003.

DELGADO, RAFAEL. Falange en Guatemala. Una amenaza para la democracia. México: Gráfica Panamericana, 1948.

Gramsci, Antonio. Cuadernos de la cárcel. México: Era, 1981.

GrijaLVA, GaBRiELA. "El Libro Negro del Comunismo en Guatemala (1954) como aproximación a la lucha de paradigmas de la categoría patria". Estudios Digital 7.18 (2019): 1-41.

JunTa DE Gobierno. Estatuto Político de la República de Guatemala. S/N10/08/1954, Recopilación de Leyes de Guatemala. LXXIII (1954): 52.

LEÓN XIII. "Rerum novarum. Sobre la situación de los obreros". Doctrina Social de la Iglesia. 18 mensajes sociales. Guatemala: Universidad Rafael Landívar. 1992. 2-26.

ORGANIZACIÓN DE ESTADOS AMERICANOS. "Declaración Americana de los Derechos y Deberes del Hombre". Colombia, 1948.

OrganizaCión De Estados Americanos, Carta de la Organización de los Estados Americanos. Bogotá, 1948.

OtAROla Aqueveque, HumberTo. "Algunos comentarios sobre la 'Declaración de Solidaridad para la Preservación de la Integridad Política de los 
Estados Americanos Contra la Intervención del Comunismo Internacional"'. Revista de Derecho 96.xXIV (1956): 173-191.

PATTO sá MotTA, RodRigo. Em guarda contra o perigo vermelho: o anticomunismo no Brasil. São Paulo: Facultad de Filosofía y Letras y Ciencias Humanas-Universidad de Sao Paulo, 2000.

PetTinà, VANni. Historia mínima de la Guerra Fría en América Latina. México: El Colegio de México, 2018.

PERUTKA, LuKÁŠs. "Arms for Árbenz. Czechoslovakia's Involvement in the Cold War in Latin America". Central European Journal of International E Security Studies 7.3 (2013): 59-76.

PIO X. "Divini Redemptoris. Sobre el comunismo ateo". Doctrina Social de la Iglesia. 18 mensajes sociales, Guatemala: Universidad Rafael Landívar (1992): 66-90.

Prieto Laurens, Jorge. Cincuenta años de Política Mexicana. Memorias Políticas. México: Editora Mexicana de Periódicos, Libros y Revistas, 1968.

ROSELl y ARELLANO, Mariano. Sobre la amenaza comunista en nuestra patria. Guatemala: Conferencia Episcopal de Guatemala, 1945.

Rosell y ARELlano, Mariano. Sobre los avances del comunismo. Guatemala: Acción Católica Guatemalteca, 1954.

Santiago Jiménez, Mario Virgilio. "Entre "hisPanistas" y "PRO-Yanquis". El Primer Congreso contra la Intervención Soviética en América Latina. 1954. Artículo en línea disponible https://journals.openedition. org/nuevomundo/70497\#bodyftn1 (consultado el 12 de noviembre de 2019).

Secretaría de Divulgación, Cultura y Turismo de la Presidencia de la RepúBlicA. Así se gestó la liberación. Guatemala: Publicaciones de la Secretaría de Divulgación, Cultura y Turismo de la Presidencia de la República, 1956.

Secretaría General de la Comisión Permanente del Primer Congreso CONTRA LA INTERVENCIÓN SOVIÉTICA EN AMÉRICA LATINA. El libro negro de comunismo en Guatemala. México: Turanzas del Valle, 1954.

TARACENA ARRIOLA, ARTURO. Etnicidad, Estado y nación en Guatemala: 1808-1944, vol. I. Guatemala: CIRMA/Nawal Wuj, 2002. 
VÁzQuez Medeles, Juan CaRlos. "La presencia guatemalteca en los Congresos anticomunistas latinoamericanos (1954-1980)". Cuadernos de Inter.c.a.mbio sobre Centroamérica y el Caribe 17.2 (2020): 1-28.

ARCHIVOS CONSULTADOS

Archivo Histórico Genaro Estrada-Acervo Histórico Diplomático, Secretaría de Relaciones Exteriores, México.

Archivo Histórico de la Policía Nacional, Guatemala.

Freedom of Information Act, Electronic Reading Room, Central Intelligence Agency.

\section{HEMEROGRAFÍA}

"El Congreso Antisoviético se convirtió en mitote". El Informador. México, 30 de mayo de 1954c. 1 y 3.

"El terror rojo invade a Guatemala". El Heraldo. México, 14 de febrero de 1954. 1 y 4 .

"Se inicia hoy un Congreso Anticomunista en México". El Informador. México, 27 de mayo de 1954a. 1 y 3.

"De un momento a otro estalla una revolución en Guatemala". El Informador. México, 28 de mayo de 1954b. 1. 